\title{
Dynamics and chaos control in a discrete-time ratio-dependent Holling-Tanner model
}

Sarker Md. Sohel Rana

Correspondence:

srana.mthdu@gmail.com

'Department of Mathematics,

University of Dhaka, Dhaka 1000,

Bangladesh

\begin{abstract}
A discrete-time Holling-Tanner model with ratio-dependent functional response is examined. We show that the system experiences a flip bifurcation and Neimark-Sacker bifurcation or both together at positive fixed point in the interior of $\mathbb{R}_{+}^{2}$ when one of the model parameter crosses its threshold value. We concentrate our attention to determine the existence conditions and direction of bifurcations via center manifold theory. To validate analytical results, numerical simulations are employed which include bifurcations, phase portraits, stable orbits, invariant closed circle, and attracting chaotic sets. In addition, the existence of chaos in the system is justified numerically by the sign of maximum Lyapunov exponents and fractal dimension. Finally, we control chaotic trajectories exists in the system by feedback control strategy.
\end{abstract}

Keywords: Holling-Tanner model, Ratio-dependent, Bifurcations, Chaos, Feedback control

MSC: (2010) 39A33, 37D45, 39A28, 92B05

\section{Introduction}

Mathematical modeling is a promising approach to understand and analyze the dynamics of ecological systems. In population ecology, the classical and significant studied theme is the interaction between predator and prey species. In recent year, the Leslie type predator-prey model has received more interest to investigate the dynamical behaviors between the species. The dynamic complexity of predator-prey system depends on predator's functional response. Holling type II functional response is mostly used functional response among arthropod predators. The Leslie predator-prey model with Holling type II functional response is called Holling-Tanner model. A number of famous ecologist and mathematician have been given attention and investigated extensively Holling-Tanner models [1-3]. Their empirical works found complex dynamical behaviors including stable or unstable limit cycle, stability states around positive equilibrium. They showed that the asymptotic stability of the positive equilibrium does not imply the global stability. They also showed that the model may undergo the Bogdanov-Takens bifurcation and the subcritical Hopf bifurcation if parameters vary in a small vicinity of critical values. But Holling-Tanner models with ratio-dependent functional response (1) has been studied in [4]. The authors established the global stability conditions of the model near positive equilibrium with the help of Lyapunov function. It is also proved that the model (1) possess a

(c) The author(s). 2019 Open Access This article is distributed under the terms of the Creative Commons Attribution 4.0 International License (http://creativecommons.org/licenses/by/4.0/), which permits unrestricted use, distribution, and reproduction in any medium, provided you give appropriate credit to the original author(s) and the source, provide a link to the Creative Commons license, and indicate if changes were made. 
unique stable limit cycle at the positive equilibrium showing coexistence of predator and prey in the sense of oscillatory balance behavior.

However, lots of exploratory works have recommend that if population size is small, or population generations are relatively discrete (nonoverlapping), or population changes within certain time intervals, one can consider the difference equation model rather than differential equation model to reveal chaotic dynamics [5-15]. These researches explored many complex properties including flip and Neimark-Sacker bifurcations, stable orbits and chaotic attractors which had been derived either by numerically or by normal form and center manifold theory.

Recently, a little works in literature studied discrete-time Holling-Tanner models [16-18] and its chaotic behaviors. For instance, a discrete-time Holling and Leslie type predator-prey system with constant-yield prey harvesting analyzed in [16], in [17] the authors investigated a discrete Holling-Tanner model and a discrete predator-prey model with modified Holling-Tanner functional response discussed in [18]. These studies paid their attention to determine the stability and directions of flip and Neimark-Sacker bifurcations via use of center manifold theory.

The following ratio-dependent Holling-Tanner model [4] is considered in this paper:

$$
\begin{aligned}
& \dot{x}=r x\left(1-\frac{x}{K}\right)-\frac{m x}{x+A y} y \\
& \dot{y}=s y\left(1-h \frac{y}{x}\right)
\end{aligned}
$$

where $x$ and $y$ represent prey and predator population densities, respectively; $r$ and $s$ are the intrinsic growth rates of the prey and predator, respectively. $K$ is the prey environment carrying capacity. The predator consumes prey according to the ratio-dependent Holling type II functional response $\frac{m x}{x+A y}$. The carrying capacity $x / h$ of predator is proportional to the population size of the prey. The parameter $h$ is the number of preys required to support predator births. $m$ is the maximal predator per capita consumption rate; $A$ is the number of prey necessary to achieve one-half of the maximum rate $m$; the constants $r, K, A, m, s$ and, $h$ all are being positive.

We introduce the new variables and parameters by the following scaling transformations:

$$
\frac{x}{K} \rightarrow x, \frac{m y}{r K} \rightarrow y, r t \rightarrow t \text { and, } a=\frac{r A}{m}, d=\frac{s h}{m}, b=\frac{m}{h r} .
$$

Then, the system (1) becomes

$$
\begin{aligned}
& \dot{x}=x(1-x)-\frac{x y}{x+a y} \\
& \dot{y}=d y\left(b-\frac{y}{x}\right)
\end{aligned}
$$

To get following two-dimensional discrete system, forward Euler scheme with integral step size $\delta$ is applied to system (2):

$$
\left(\begin{array}{l}
x \\
y
\end{array}\right) \mapsto\left(\begin{array}{c}
x+\delta x\left[(1-x)-\frac{y}{x+a y}\right] \\
y+\delta y\left[d\left(b-\frac{y}{x}\right)\right]
\end{array}\right)
$$

This study will mainly focus on how model parameters affect on the dynamics of system (3). Especially, we discuss systematically to show the existence condition of Flip bifurcation and Neimark-Sacker bifurcation using bifurcation theory and center manifold theory [19]. 
Because in the discrete predator-prey system, Flip bifurcation and Neimark-Sacker bifurcation both are the important mechanisms for the generation of complex dynamics and both bifurcations cause the system to jump from stable window to chaotic states through periodic and quasi-periodic states, and trigger a route to chaos.

The outlines of this paper is as follows. The existence condition for fixed points of system (3) in the interior of $\mathbb{R}_{+}^{2}$ and their stability analysis are given in "Existence conditions and stability analysis of fixed points" section. In "Direction and stability analysis of bifurcation" section, we determine direction of bifurcation for system (3) under certain parametric condition. The bifurcation diagrams, phase portraits, maximum Lyapunov exponents, and Fractal dimensions are presented numerically in "Numerical simulations" section by changing one or more control parameters values. In "Controlling chaos" section, a feedback control technique has been used to stabilize unstable trajectories. A short conclusion is presented in the "Discussions" section.

\section{Existence conditions and stability analysis of fixed points}

To find fixed points of system (3), we set

$$
\left\{\begin{array}{l}
x+\delta x\left[(1-x)-\frac{y}{x+a y}\right]=x \\
y+\delta y\left[d\left(b-\frac{y}{x}\right)\right]=y
\end{array}\right.
$$

By solving system of non-linear Eqs. 4 we obtain the following result.

Lemma 1 For all feasible values of parameters, the system (3)

(i) always has axial fixed point $E_{1}(1,0)$,

(ii) has a unique coexistence fixed point $E_{2}\left(x^{*}, y^{*}\right)$ if $b<1+a b$ where $x^{*}=$ $\frac{1-b+a b}{1+a b}$ and $y^{*}=b x^{*}$.

Next, we analyze local stability at each fixed points for system (3). The Jacobian matrix of system (3) around arbitrary fixed point $E(x, y)$ is given by

$$
J(x, y)=\left(\begin{array}{ll}
j_{11} & j_{12} \\
j_{21} & j_{22}
\end{array}\right)
$$

where

$$
\begin{aligned}
& j_{11}=1+\delta\left(1-2 x+\frac{x y}{(x+a y)^{2}}-\frac{y}{x+a y}\right), j_{12}=\delta\left(\frac{a x y}{(x+a y)^{2}}-\frac{x}{x+a y}\right) \\
& j_{21}=\frac{d \delta y^{2}}{x^{2}}, j_{22}=1-\frac{d \delta y}{x}+d \delta\left(b-\frac{y}{x}\right) .
\end{aligned}
$$

The characteristic equation of matrix $J$ is

$$
\lambda^{2}+p(x, y) \lambda+q(x, y)=0
$$

where $p(x, y)=-\operatorname{trJ}=-\left(j_{11}+j_{22}\right)$ and $\operatorname{det} J=j_{11} j_{22}-j_{12} j_{21}$.

Now, the topological classification of stability around fixed points by using Jury's criterion [20] are expressed as follows.

Proposition 1 For predator free fixed point $E_{1}(1,0)$, the following topological classification true

(i) $E_{1}$ is a saddle if $0<\delta<2$, 
(ii) $E_{1}$ is a source if $\delta>2$,

(iii) $E_{1}$ is a non-hyperbolic if $\delta=2$.

Let

$$
F B_{E_{1}}=\{(a, b, d, \delta): \delta=2, a, b, d>0\} .
$$

It is obvious that when parameters are in $F B_{E_{1}}$, then one of the eigenvalues of $J\left(E_{1}\right)$ : $\lambda_{1}=1-\delta$ and $\lambda_{2}=1+b d \delta$ is -1 and the other is not equal to \pm 1 . Therefore, system (3) experience a flip bifurcation when parameters change in small vicinity of $F B_{E_{1}}$.

At $E_{2}\left(x^{*}, y^{*}\right)$, the Eq. 6 becomes

$$
F(\lambda):=\lambda^{2}-(2+L \delta) \lambda+\left(1+L \delta+M \delta^{2}\right)=0,
$$

where

$$
\begin{aligned}
& L=1+b d-2 x^{*}-\frac{2 d y^{*}}{x^{*}}-\frac{a y^{* 2}}{\left(x^{*}+a y^{* 2}\right.}, \\
& M=d\left(b-2 b x^{*}+4 y^{*}\right)-\frac{2 d y^{*}}{x^{*}}-\frac{(2+a b) d y^{*}}{\left(x^{*}+a y^{*}\right)^{2}}+\frac{\left(3 x^{*}+2 a y^{*}\right) d y^{* 2}}{x^{*}\left(x^{*}+a y^{*}\right)^{2}} .
\end{aligned}
$$

Therefore, $F(1)=M \delta^{2}>0$ and $F(-1)=4+2 L \delta+M \delta^{2}$.

For topological classification of $E_{2}$, we state following Proposition.

Proposition 2 Suppose $b<1+a b$ holds. Then for coexistence fixed point $E_{2}\left(x^{*}, y^{*}\right)$ of system (3), the following topological classification true

(i) $E_{2}$ is a sink if one of the following conditions holds

(i.1) $L^{2}-4 M \geq 0$ and $\delta<\frac{-L-\sqrt{L^{2}-4 M}}{M}$;

(i.2) $L^{2}-4 M<0$ and $\delta<-\frac{L}{M}$;

(ii) $E_{2}$ is a source if one of the following conditions holds

(ii.1) $L^{2}-4 M \geq 0$ and $\delta>\frac{-L+\sqrt{L^{2}-4 M}}{M}$;

(ii.2) $L^{2}-4 M<0$ and $\delta>-\frac{L}{M}$;

(iii) $E_{2}$ is a non-hyperbolic if one of the following conditions holds

(iii.1) $L^{2}-4 M \geq 0$ and $\delta=\frac{-L \pm \sqrt{L^{2}-4 M}}{M}$;

(iii.2) $L^{2}-4 M<0$ and $\delta=-\frac{L}{M}$;

(iv) $E_{2}$ is a saddle if otherwise.

From Proposition 2, it can be easily seen that if condition (iii.1) holds then eigenvalues of $J\left(E_{2}\right)$ are $\lambda_{1}=-1$ and $\lambda_{2} \neq \pm 1$. If (iii.2) is true, then eigenvalues of $J\left(E_{2}\right)$ are complex having modulus one.

Let

$$
F B_{E_{2}}^{1}=\left\{(a, b, d, \delta): \delta=\frac{-L-\sqrt{L^{2}-4 M}}{M}, L^{2}-4 M \geq 0, a, b, d>0\right\},
$$

or

$$
F B_{E_{2}}^{2}=\left\{(a, b, d, \delta): \delta=\frac{-L+\sqrt{L^{2}-4 M}}{M}, L^{2}-4 M \geq 0, a, b, d>0\right\} .
$$

Then system (3) experience a flip bifurcation at $E_{2}$ when parameters $(a, b, d, \delta)$ vary in a small vicinity of either set $F B_{E_{2}}^{1}$ or set $F B_{E_{2}}^{2}$. 
Also let

$$
\mathrm{NSB}_{E_{2}}=\left\{(a, b, d, \delta): \delta=-\frac{L}{M}, L^{2}-4 M<0, a, b, d>0\right\},
$$

then system (3) experience a NS bifurcation at $E_{2}$ if the parameters $(a, b, d, \delta)$ change around the set $\mathrm{NSB}_{E_{2}}$.

\section{Direction and stability analysis of bifurcation}

Here, we will pay attention to determine the direction and stability of flip bifurcation and Neimark-Sacker bifurcation of system (3) around $E_{2}$ via application of center manifold theory [19]. The integral step size $\delta$ is being taken as a real bifurcation parameter.

\section{Flip bifurcation: direction and stability}

We take the parameters $(a, \alpha, \beta, \delta)$ arbitrarily locate in $\mathrm{FB}_{E_{2}}^{1}$. For other set $\mathrm{FB}_{E_{2}}^{2}$, one can apply similar reasoning. Consider the system (3) at fixed point $E_{2}\left(x^{*}, y^{*}\right)$ with parameters lie in $\mathrm{FB}_{E_{2}}^{1}$.

Let

$$
\delta=\delta_{F}=\frac{-L-\sqrt{L^{2}-4 M}}{M},
$$

then the eigenvalues of $J\left(E_{2}\right)$ are

$$
\lambda_{1}\left(\delta_{F}\right)=-1 \text { and } \lambda_{2}\left(\delta_{F}\right)=3+L \delta_{F} .
$$

In order for $\left|\lambda_{2}\left(\delta_{F}\right)\right| \neq 1$, we have

$$
L \delta_{F} \neq-2,-4 \text {. }
$$

Assume that $\tilde{x}=x-x^{*}, \quad \tilde{y}=y-y^{*}$, and set $A(\delta)=J\left(x^{*}, y^{*}\right)$. Then, we transform the fixed point $\left(x^{*}, y^{*}\right)$ of system (3) to the origin. By Taylor expansion, system (3) can be written as

$$
\left(\begin{array}{c}
\tilde{x} \\
\tilde{y}
\end{array}\right) \rightarrow A(\delta)\left(\begin{array}{l}
\tilde{x} \\
\tilde{y}
\end{array}\right)+\left(\begin{array}{c}
F_{1}(\tilde{x}, \tilde{y}, \delta) \\
F_{2}(\tilde{x}, \tilde{y}, \delta)
\end{array}\right)
$$

where $X=(\tilde{x}, \tilde{y})^{T}$ is the vector of the transformed system and

$$
\begin{aligned}
& F_{1}(\tilde{x}, \tilde{y}, \delta)=\frac{1}{6} {\left[-\frac{6 a \delta y^{* 2}}{\left(x^{*}+a y^{*}\right)^{4}} \tilde{x}^{3}-\frac{6 a^{2} \delta x^{* 2}}{\left(x^{*}+a y^{*}\right)^{4}} \tilde{x}^{3}-\frac{6 a \delta y^{*}\left(-2 x^{*}+a y^{*}\right)}{\left(x^{*}+a y^{*}\right)^{4}} \tilde{x}^{2} \tilde{y}+\frac{6 a \delta x^{*}\left(-x^{*}+2 a y^{*}\right)}{\left(x^{*}+a y^{*}\right)^{4}} \tilde{x} \tilde{y}^{2}\right] } \\
&+\frac{1}{2}\left[\delta\left(-2-\frac{2 x^{*} y^{*}}{\left(x^{*}+a y^{*}\right)^{3}}+\frac{2 y^{*}}{\left(x^{*}+a y^{*}\right)^{2}}\right) \tilde{x}^{2}+\frac{2 a \delta x^{* 2}}{\left(x^{*}+a y^{*}\right)^{3}} \tilde{y}^{2}-\frac{4 a \delta x^{*} y^{*}}{\left(x^{*}+a y^{*}\right)^{3}} \tilde{x} \tilde{y}\right] \\
& \quad+O\left(\|X\|^{4}\right) \\
& F_{2}(\tilde{x}, \tilde{y}, \delta)=\frac{d \delta}{x^{* 4}} \tilde{x}\left(x^{*} \tilde{y}-y^{*} \tilde{x}\right)^{2}-\frac{d \delta}{x^{* 3}} \tilde{x}\left(x^{*} \tilde{y}-y^{*} \tilde{x}\right)^{2}+O\left(\|X\|^{4}\right)
\end{aligned}
$$

The system (8) can be expressed as $X_{n+1}=A X_{n}+\frac{1}{2} B\left(X_{n}, X_{n}\right)+\frac{1}{6} C\left(X_{n}, X_{n}, X_{n}\right)+O\left(X_{n}^{4}\right)$ where $B(x, y)=\left(\begin{array}{l}B_{1}(x, y) \\ B_{2}(x, y)\end{array}\right)$ and $C(x, y, u)=\left(\begin{array}{l}C_{1}(x, y, u) \\ C_{2}(x, y, u)\end{array}\right)$ are symmetric multi-linear vector functions of $x, y, u \in \mathbb{R}^{2}$ and defined as follows: 


$$
\begin{aligned}
B_{1}(x, y)= & \left.\sum_{j, k=1}^{2} \frac{\delta^{2} F_{1}(\xi, \delta)}{\delta \xi_{j} \delta \xi_{k}}\right|_{\xi=0} x_{j} y_{k}=-\frac{2 a \delta x^{*} y^{*}}{\left(x^{*}+a y^{*}\right)^{3}}\left(x_{1} y_{2}+x_{2} y_{1}\right)+\frac{2 a \delta x^{* 2}}{\left(x^{*}+a y^{*}\right)^{3}} x_{2} y_{2} \\
& +\delta\left(-2-\frac{2 x^{*} y^{*}}{\left(x^{*}+a y^{*}\right)^{3}}+\frac{2 y^{*}}{\left(x^{*}+a y^{*}\right)^{2}}\right) x_{1} y_{1}, \\
B_{2}(x, y)= & \left.\sum_{j, k=1}^{2} \frac{\delta^{2} F_{2}(\xi, \delta)}{\delta \xi_{j} \delta \xi_{k}}\right|_{\xi=0} x_{j} y_{k}=-\frac{2 d \delta}{x^{*}} x_{2} y_{2}+\frac{2 d \delta y^{*}}{x^{* 2}}\left(x_{1} y_{2}+x_{2} y_{1}\right)-\frac{2 d \delta y^{* 2}}{x^{* 3}} x_{1} y_{1}, \\
C_{1}(x, y, u)= & \left.\sum_{j, k, l=1}^{2} \frac{\delta^{2} F_{1}(\xi, \delta)}{\delta \xi_{j} \delta \xi_{k} \delta \xi_{l}}\right|_{\xi=0} x_{j} y_{k} u_{l}=-\frac{6 a \delta y^{* 2}}{\left(x^{*}+a y^{*}\right)^{4}} x_{1} y_{1} u_{1}-\frac{6 a^{2} \delta x^{* 2}}{\left(x^{*}+a y^{*}\right)^{4}} x_{2} y_{2} u_{2} \\
& \quad-\frac{2 a y^{*}\left(-2 x^{*}+a y^{*}\right) \delta}{\left(x^{*}+a y^{*}\right)^{4}}\left(x_{1} y_{2} u_{1}+x_{2} y_{1} u_{1}+x_{1} y_{1} u_{2}\right) \\
& +\frac{2 a x^{*}\left(-x^{*}+2 a y^{*}\right) \delta}{\left(x^{*}+a y^{*}\right)^{4}}\left(x_{1} y_{2} u_{2}+x_{2} y_{1} u_{2}+x_{2} y_{2} u_{1}\right), \\
C_{2}(x, y, u)= & \left.\sum_{j, k, l=1}^{2} \frac{\delta^{2} F_{2}(\xi, \delta)}{\delta \xi_{j} \delta \xi_{k} \delta \xi_{l}}\right|_{\xi=0} x_{j} y_{k} u_{l}=\frac{2 d \delta}{x^{* 2}}\left(x_{1} y_{2} u_{2}+x_{2} y_{1} u_{2}+x_{2} y_{2} u_{1}\right) \\
& \quad-\frac{4 d \delta y^{*}}{x^{* 3}}\left(x_{1} y_{1} u_{2}+x_{1} y_{2} u_{1}+x_{2} y_{1} u_{1}\right)+\frac{6 d \delta y^{* 2}}{x^{* 4}} x_{1} y_{1} u_{1} .
\end{aligned}
$$

Let $p, q \in \mathbb{R}^{2}$ be eigenvectors of $A$ and transposed matrix $A^{T}$ respectively for $\lambda_{1}\left(\delta_{F}\right)=$ -1 Then, we have

$$
A\left(\delta_{F}\right) q=-q \quad \text { and } \quad A^{T}\left(\delta_{F}\right) p=-p .
$$

Direct computation shows

$$
\begin{aligned}
& q \sim\left(2+b d \delta_{F}-\frac{2 d \delta_{F} y^{*}}{x^{*}},-\frac{d \delta_{F} y^{* 2}}{x^{* 2}}\right)^{T}, \\
& p \sim\left(2+b d \delta_{F}-\frac{2 d \delta_{F} y^{*}}{x^{*}}, \frac{\delta_{F} x^{* 2}}{\left(x^{*}+a y^{*}\right)^{2}}\right)^{T} .
\end{aligned}
$$

We use $\langle p, q\rangle=p_{1} q_{1}+p_{2} q_{2}$, standard scalar product in $\mathbb{R}^{2}$ to normalize the vectors $p$ and $q$. Setting the normalized vectors as

$$
\begin{aligned}
& q=\left(2+b d \delta_{F}-\frac{2 d \delta_{F} y^{*}}{x^{*}},-\frac{d \delta_{F} y^{* 2}}{x^{* 2}}\right)^{T}, \\
& p=\gamma_{1}\left(2+b d \delta_{F}-\frac{2 d \delta_{F} y^{*}}{x^{*}}, \frac{\delta_{F} x^{* 2}}{\left(x^{*}+a y^{*}\right)^{2}}\right)^{T} .
\end{aligned}
$$

where $\gamma_{1}=\frac{1}{\left(2+b d \delta_{F}-\frac{2 d \delta_{F} y^{*}}{x^{*}}\right)^{2}-\frac{d \delta_{F}^{2} y^{* 2}}{\left(x^{*}+a y^{*}\right)^{2}}}$. We see that $\langle p, q\rangle=1$.

The sign of coefficient $l_{1}\left(\delta_{F}\right)$ determines the direction of flip bifurcation and is computed by

$$
l_{1}\left(\delta_{F}\right)=\frac{1}{6}\langle p, C(q, q, q)\rangle-\frac{1}{2}\left\langle p, B\left(q,(A-I)^{-1} B(q, q)\right)\right\rangle
$$

We summarize above discussion into the following theorem for direction and stability of flip bifurcation. 
Theorem 1 Assume that (7) holds. Then, if $l_{1}\left(\delta_{F}\right) \neq 0$ and the parameter $\delta$ varies its value in a small vicinity of $F B_{E_{2}}^{1}$, the system (3) experiences a flip bifurcation at positive fixed point $E_{2}\left(x^{*}, y^{*}\right)$. Moreover, if $l_{1}\left(\delta_{F}\right)>0$ (resp., $\left.l_{1}\left(\delta_{F}\right)<0\right)$, then there exists stable (resp., unstable) period-2 orbits bifurcate from $E_{2}\left(x^{*}, y^{*}\right)$.

\section{Neimark-Sacker bifurcation: direction and stability}

Next, we take the parameters $(a, \alpha, \beta, \delta)$ arbitrarily located in $\mathrm{NSB}_{E_{2}}$. Consider the system (3) at fixed point $E_{2}\left(x^{*}, y^{*}\right)$ with parameters vary in the vicinity of $\mathrm{NSB}_{E_{2}}$. Then, the roots (eigenvalues) of Eq. 6 are pair of complex conjugate and given by

$$
\lambda, \bar{\lambda}=\frac{-p(\delta) \pm i \sqrt{4 q(\delta)-p(\delta)^{2}}}{2}=1+\frac{L \delta}{2} \pm \frac{i \delta}{2} \sqrt{4 M-L^{2}} .
$$

Let

$$
\delta=\delta_{\mathrm{NS}}=-\frac{L}{M}
$$

Then, we have $|\lambda|=\sqrt{q\left(\delta_{\mathrm{NS}}\right)}=1$.

From the transversality condition, we get

$$
\left.\frac{d|\lambda(\delta)|}{d \delta}\right|_{\delta=\delta_{\mathrm{NS}}}=-\frac{L}{2} \neq 0
$$

Moreover, the nonresonance condition $p\left(\delta_{\mathrm{NS}}\right) \neq 0,1$ obviously satisfies

$$
\frac{L^{2}}{M} \neq 2,3
$$

and we have

$$
\lambda^{k}\left(\delta_{\mathrm{NS}}\right) \neq 1 \text { for } k=1,2,3,4
$$

Let $q, p \in \mathbb{C}^{2}$ be eigenvectors of $A\left(\delta_{\mathrm{NS}}\right)$ and $A^{T}\left(\delta_{\mathrm{NS}}\right)$ for eigenvalues $\lambda\left(\delta_{\mathrm{NS}}\right)$ and $\bar{\lambda}\left(\delta_{\mathrm{NS}}\right)$ respectively such that

$$
A\left(\delta_{\mathrm{NS}}\right) q=\lambda\left(\delta_{\mathrm{NS}}\right) q, \quad A\left(\delta_{\mathrm{NS}}\right) \bar{q}=\bar{\lambda}\left(\delta_{\mathrm{NS}}\right) \bar{q}
$$

and

$$
A^{T}\left(\delta_{\mathrm{NS}}\right) p=\bar{\lambda}\left(\delta_{\mathrm{NS}}\right) p, \quad A^{T}\left(\delta_{\mathrm{NS}}\right) \bar{p}=\lambda\left(\delta_{\mathrm{NS}}\right) \bar{p} .
$$

By direct calculation, we obtain

$$
\begin{aligned}
& q \sim\left(1+b d \delta_{\mathrm{NS}}-\frac{2 d \delta_{\mathrm{NS}} y^{*}}{x^{*}}-\lambda,-\frac{d \delta_{\mathrm{NS}} y^{* 2}}{x^{* 2}}\right)^{T}, \\
& p \sim\left(1+b d \delta_{\mathrm{NS}}-\frac{2 d \delta_{\mathrm{NS}} y^{*}}{x^{*}}-\bar{\lambda}, \frac{\delta_{\mathrm{NS}} x^{*}}{\left(x^{*}+a y^{*}\right)^{2}}\right)^{T} .
\end{aligned}
$$

For normalization of the vectors $p$ and $q$, we set $p=\gamma_{2}$ $\left(1+b d \delta_{\mathrm{NS}}-\frac{2 d \delta_{\mathrm{NS}} y^{*}}{x^{*}}-\bar{\lambda}, \frac{\delta_{\mathrm{NS}} x^{*}}{\left(x^{*}+a y^{*}\right)^{2}}\right)^{T}$ where

$$
\gamma_{2}=\frac{1}{\left(1+b d \delta_{\mathrm{NS}}-\frac{2 d \delta_{\mathrm{NS}} y^{*}}{x^{*}}-\bar{\lambda}\right)^{2}-\frac{d \delta_{\mathrm{NS}}^{2} x^{* 2} y^{* 2}}{x^{*}\left(x^{*}+a y^{*}\right)^{2}}} .
$$

Then we see that $\langle p, q\rangle=\overline{p_{1}} q_{2}+\overline{p_{2}} q_{1}=1$. 
When $\delta$ close to $\delta_{\mathrm{NS}}$ and $z \in \mathbb{C}$, the vector $X \in \mathbb{R}^{2}$ can be decomposed uniquely as $X=z q+\bar{z} \bar{q}$.

It is obvious that $z=\langle p, X\rangle$. Thus, we obtain the following transformed form of system (8) for all sufficiently small $|\delta|$ near $\delta_{\mathrm{NS}}$ :

$$
z \mapsto \lambda(\delta) z+g(z, \bar{z}, \delta)
$$

where $\lambda(\delta)=(1+\varphi(\delta)) e^{i \theta(\delta)}$ with $\varphi\left(\delta_{N S}\right)=0$ and $g(z, \bar{z}, \delta)$ is a smooth complex-valued function. According to Taylor expression, the function $g$ can be written as

$$
g(z, \bar{z}, \delta)=\sum_{k+l \geq 2} \frac{1}{k ! l !} g_{k l}(\delta) z^{k} \bar{z}^{l}, \text { with } g_{k l} \in \mathbb{C}, k, l=0,1, \cdots
$$

By symmetric multi-linear vector functions, the Taylor coefficients $g_{k l}$ are obtained as

$$
\begin{aligned}
& g_{20}\left(\delta_{\mathrm{NS}}\right)=\langle p, B(q, q)\rangle, \\
& g_{11}\left(\delta_{\mathrm{NS}}\right)=\langle p, B(q, \bar{q})\rangle \\
& g_{02}\left(\delta_{\mathrm{NS}}\right)=\langle p, B(\bar{q}, \bar{q})\rangle, \\
& g_{21}\left(\delta_{\mathrm{NS}}\right)=\langle p, C(q, q, \bar{q})\rangle,
\end{aligned}
$$

The coefficient $l_{2}\left(\delta_{\mathrm{NS}}\right)$ which determines the direction of Neimark-Sacker bifurcation in a generic system exhibiting invariant closed curve can be calculated via

$$
\begin{aligned}
& l_{2}\left(\delta_{\mathrm{NS}}\right)=\operatorname{Re}\left(\frac{e^{-i \theta\left(\delta_{\mathrm{NS}}\right)} g_{21}}{2}\right)-\operatorname{Re}\left(\frac{\left(1-2 e^{i \theta\left(\delta_{\mathrm{NS}}\right)}\right) e^{-2 i \theta\left(\delta_{\mathrm{NS}}\right)}}{2\left(1-e^{i \theta\left(\delta_{\mathrm{NS}}\right)}\right)} g_{20} g_{11}\right)-\frac{1}{2}\left|g_{11}\right|^{2}-\frac{1}{4}\left|g_{02}\right|^{2} \text {, where } \\
& e^{i \theta\left(\delta_{\mathrm{NS}}\right)}=\lambda\left(\delta_{\mathrm{NS}}\right) .
\end{aligned}
$$

Summarizing above analysis, we present the following theorem for direction and stability of Neimark-Sacker bifurcation.

Theorem 2 Suppose that (13) holds and $l_{2}\left(\delta_{\mathrm{NS}}\right) \neq 0$. If the parameter $\delta$ varies its value in small neighborhood of $\mathrm{NSB}_{E_{2}}$, then system (3) experiences a Neimark-Sacker bifurcation at positive fixed point $E_{2}$. Moreover, if the sign of $l_{2}\left(\delta_{\mathrm{NS}}\right)$ is negative (resp., positive), then a unique invariant closed curve bifurcates from $E_{2}$ which is attracting (resp., repelling) and the Neimark-Sacker bifurcation is supercritical (resp., subcritical).

\section{Numerical simulations}

In this section, numerical simulation are performed to validate our theoretical results, especially diagrams for bifurcation of system (3) at fixed point $E_{2}$, phase portraits, maximum Lyapunov exponents and fractal dimension corresponding to bifurcation diagrams. For bifurcation analysis, we consider different sets for parameter values as given in Table 1 :

Example 1 Flip bifurcation with bifurcation parameter $\delta$ covering $[2.6,3.86]$

Table 1 Parameter values

\begin{tabular}{lll}
\hline & Varying parameter in range & Fixed parameters \\
\hline Case (i) & $2.6 \leq \delta \leq 3.86$ & $a=3.0, b=1.0, d=0.1$ \\
Case (ii) & $2.0 \leq \delta \leq 4.5$ & $a=0.6, b=1.0, d=0.1$ \\
Case (iii) & $0.6 \leq a \leq 3.0$ & $b=1.0, d=0.1, \delta=3.9$ \\
Case (iv) & $2.0 \leq \delta \leq 4.5, \quad 0.6 \leq a \leq 3.0$ & $b=1.0, d=0.1$. \\
\hline
\end{tabular}


We set values of parameter as given in case (i). By calculation, we obtain a unique fixed point $E_{2}(0.75,0.75)$ of system (3). A flip bifurcation point is evaluated as $\delta_{F}=2.95569$. It is observed that the system (3) experiences a flip bifurcation around $E_{2}$ when $\delta$ passes its critical value $\delta_{F}$. Also, at $\delta=\delta_{F}$ the corresponding eigenvalues are $\lambda_{1}=-1, \lambda_{2}=0.672397$, $a\left(\delta_{F}\right)=20.5735$ and $(a, b, d, \delta) \in \mathrm{FB}_{E_{2}}^{1}$. This shows the correctness of Theorem 1 .

The bifurcation diagrams shown in Fig. 1a, $b$ reveal that fixed point $E_{2}$ is stable for $\delta<\delta_{F}$, loses its stability at $\delta=\delta_{F}$ and for $\delta>\delta_{F}$ there exists a period doubling phenomena leading to chaos. It is also seen that the period $-2,-4,-8$, and -16 orbits emerging for $\delta \in[2.6,3.668]$, chaotic set for $\delta \in[3.69,3.86]$ and the period -12 orbit occurs at $\delta=$ 3.7924 which is in chaotic window $\delta \in[3.69,3.86]$ causing dynamic transition from periodic behaviors to chaos. The maximum Lyapunov exponents (MLE) and fractal dimension (FD) related to Fig. 1a, b are displayed in Fig. 1c, $d$ which confirm stable, periodic, or chaotic states exists in system (3).

Example 2 Neimark-Sacker (NS) bifurcation with bifurcation parameter $\delta$ covering $[2.0,4.5]$

With the variation of parameter a, the predator-prey system (3) exhibits much richer dynamics through the emergence of NS bifurcation. We take parameters as given in case (ii). After calculation, we find a unique fixed point $E_{2}(0.375,0.375)$ of system (3). A NS bifurcation point is obtained as $\delta_{\mathrm{NS}}=2.25$. It is seen that a NS bifurcation emerges around fixed point $E_{2}$ when $\delta$ passes through $\delta_{\mathrm{NS}}$. Also, we have $\lambda, \bar{\lambda}=0.905078 \pm 0.425245 i, g_{20}=$

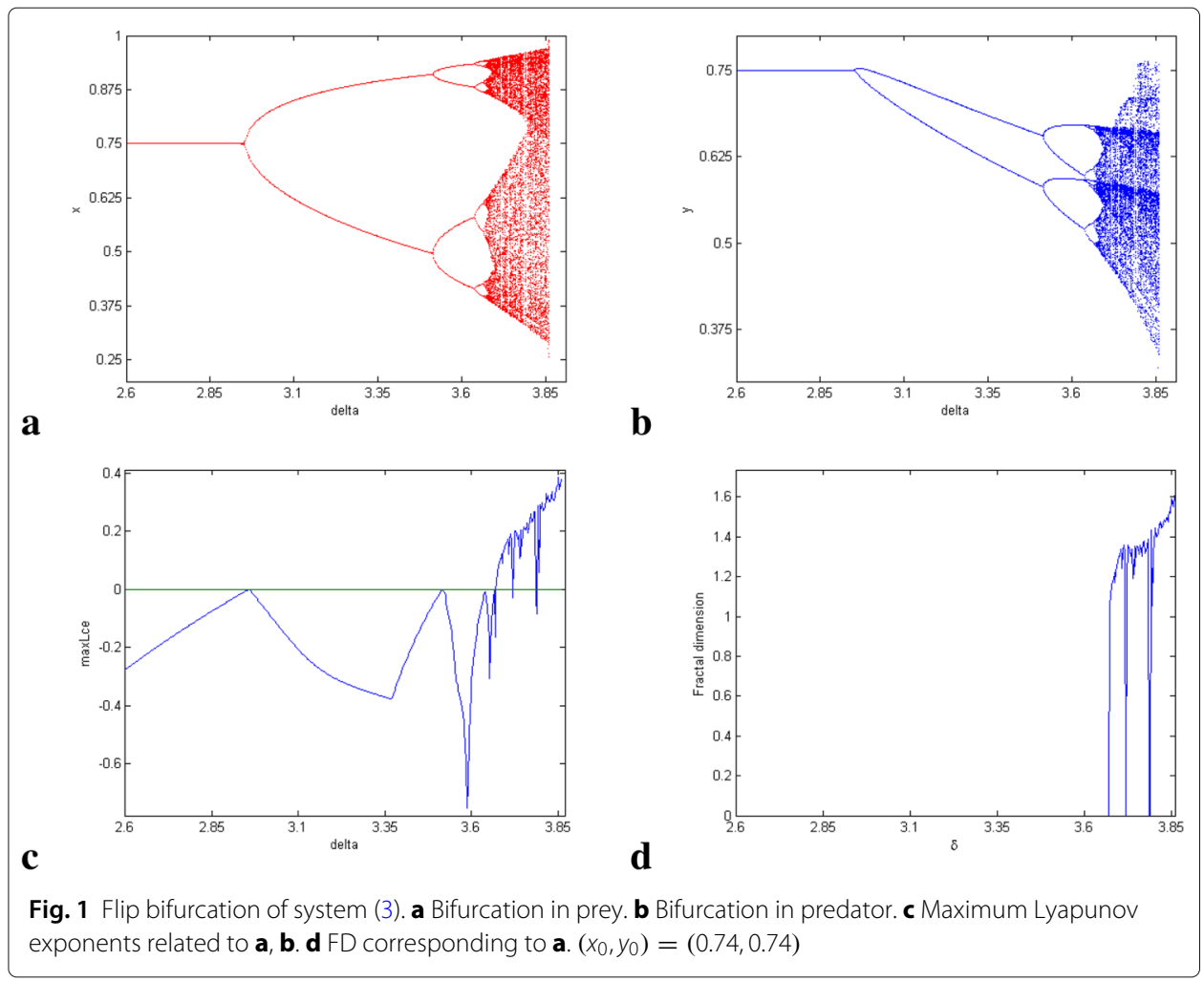


$0.359863+0.156709 i, g_{11}=0.50625-0.499099 i, g_{02}=-1.27625+0.58727 i, g_{21}=-$ $1.27332+1.64876 i, a\left(\delta_{\mathrm{NS}}\right)=-0.742379$ and $(a, b, d, \delta) \in \mathrm{NSB}_{E_{2}}$. The correctness of Theorem 2 is verified.

The bifurcation diagrams are depicted in Fig. $2 a, b$ which illustrate that the fixed point $E_{2}$ is stable for $\delta<\delta_{\mathrm{NS}}$, loses its stability near $\delta=\delta_{\mathrm{NS}}$ and an attracting invariant closed cycle appears if $\delta>\delta_{\mathrm{NS}}$. The maximum Lyapunov exponents related to Fig. $2 a, b$ are disposed in Fig. 2c, which exhibits the existences of periodic orbits and chaos as parameter $\delta$ increases. These results indicate that NS bifurcation instigates a route to chaos, through a dynamic transition from a stable state, to invariant closed cycle, with periodic and quasi-periodic states occurring in between, to chaotic sets. For instance, chaotic set is observed when $\delta \sim 4.15$ which is consistent with the sign of maximum Lyapunov exponent. Figure $2 d$ is a local amplification of Fig. 2 a for $\delta \in[3.5,4.0]$.

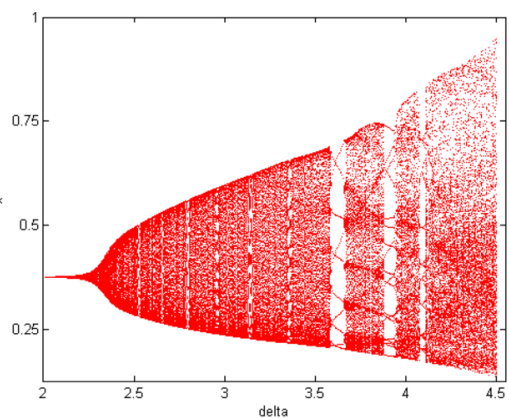

a

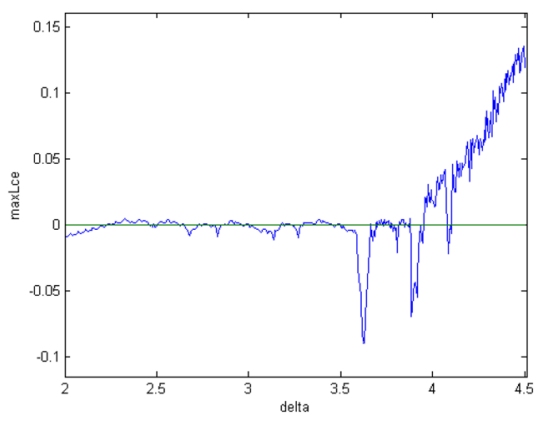

c b
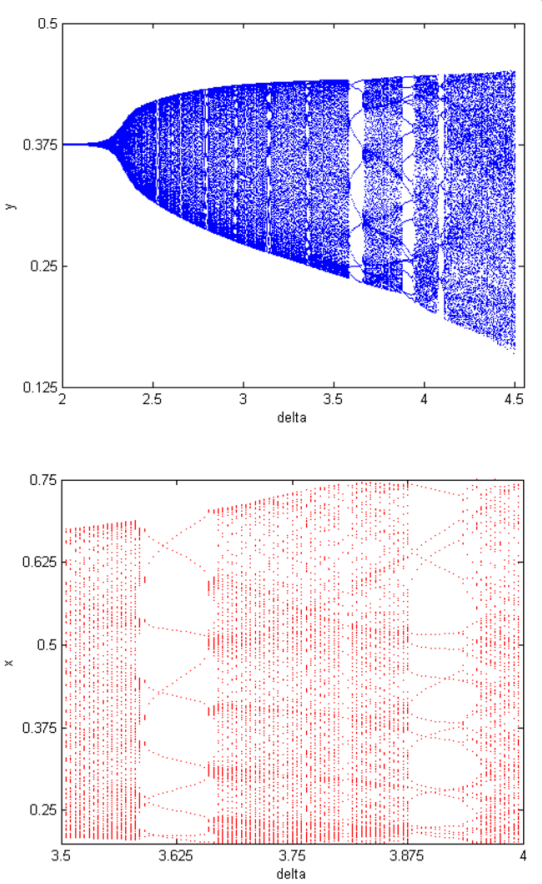

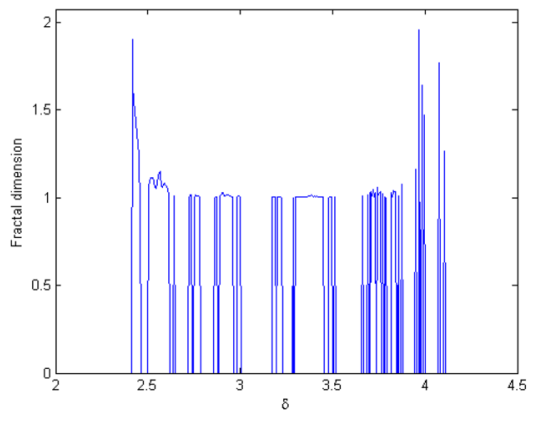

$\mathbf{e}$

Fig. 2 NS bifurcation of system (3). a NS bifurcation in prey, b NS bifurcation in predator, c maximum Lyapunov exponents corresponding to $\mathbf{a}, \mathbf{b}$. $\mathbf{d}$ Local amplification diagram in $\mathbf{a}$ for $\delta \in[3.5,4.0]$. e FD associated with a. $\left(x_{0}, y_{0}\right)=(0.37,0.37)$ 
Figure 3 explicitly shows that as the values of $\delta$ increases, there are alternation between periodic or quasi-periodic behaviors and invariant cycle or chaotic behavior. For different values of $\delta$, phase portraits of system (3) associated with Fig. $2 a, b$ are plotted in Fig. 3 illustrating the existence of period $-10,-19,-38$, and -9 orbits, and chaos in system (3) at $\delta \sim 3.6, \delta \sim 3.9, \delta \sim 3.95, \delta \sim 4.075$, and $\delta \sim 4.5$, respectively.

Example 3 Flip-NS bifurcation with bifurcation parameter a covering [0.6, 3.0]

When we set the parameter values as in case (iii), a new bifurcation diagrams is obtained as plotted in Fig. 4. This illustrates that the predator-prey system (3) experiences Neimark-Sacker bifurcation and flip bifurcation both together when parameter a passes its critical value. The system firstly enters chaotic dynamics for small value of a. However, with the increase of a value, the chaotic dynamics of the predator-prey system suddenly disappear through a NS bifurcation occurring first at a $\sim 0.691714$, and then the system dynamics jump to a stable state. Thereafter, we find that the predator-prey system undergoes a flip bifurcation occurs at $a \sim 1.92452$ and then period doubling phenomena trigger a route to chaos. The maximum Lyapunov exponents (MLE) and fractal dimension (FD) related to Fig. $4 a, b$ are displayed in Fig. $4 c, d$ which confirm dynamic transition in system (3) from chaotic set to stable window and vice versa.

Example 4 Bifurcation for parameters $\delta$ and $a$

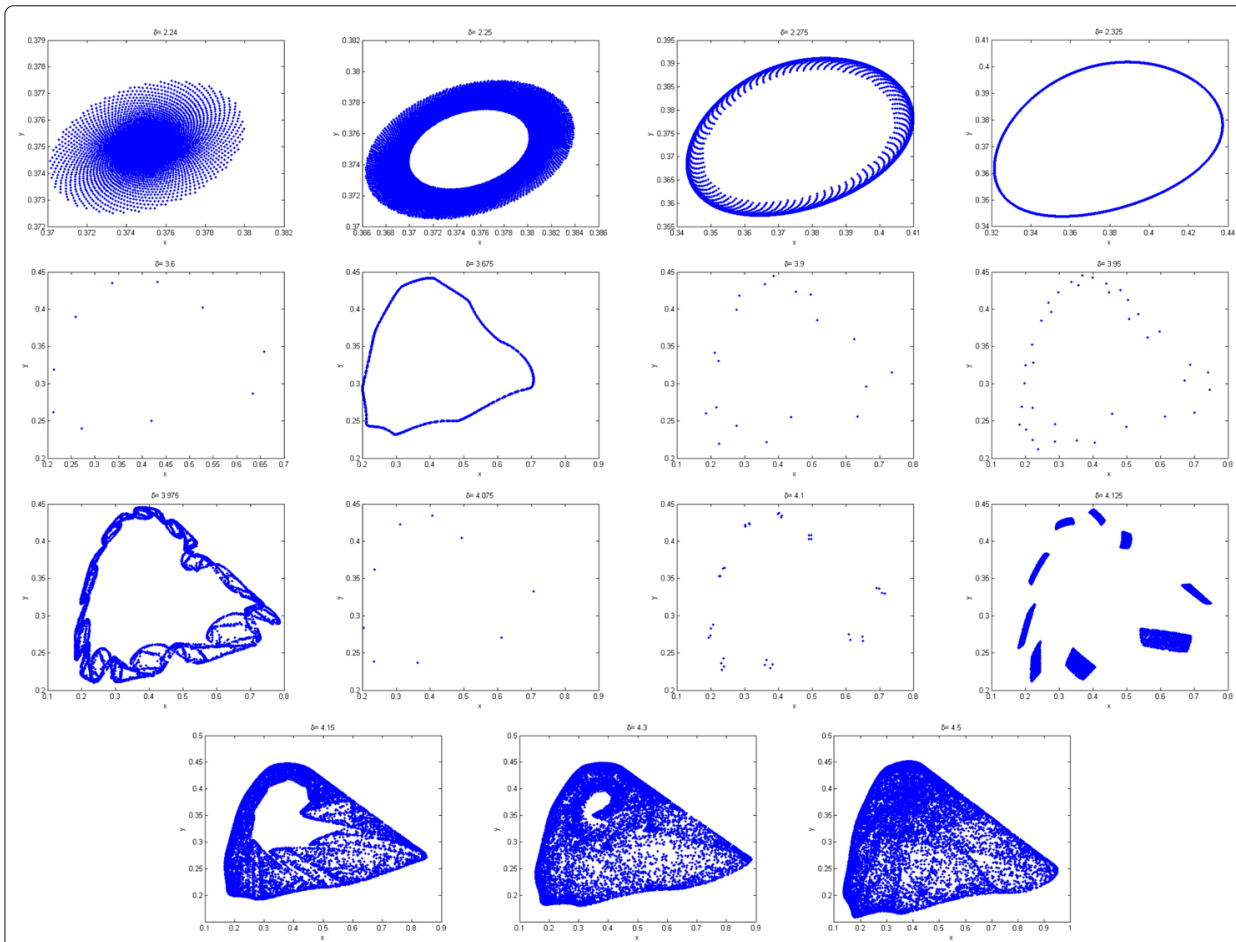

Fig. 3 Phase portraits for different values of $\delta$ corresponding to Fig. 2a, $\mathbf{b}$ 


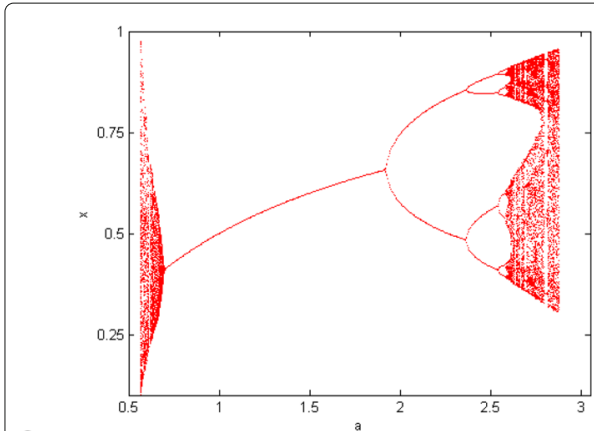

a

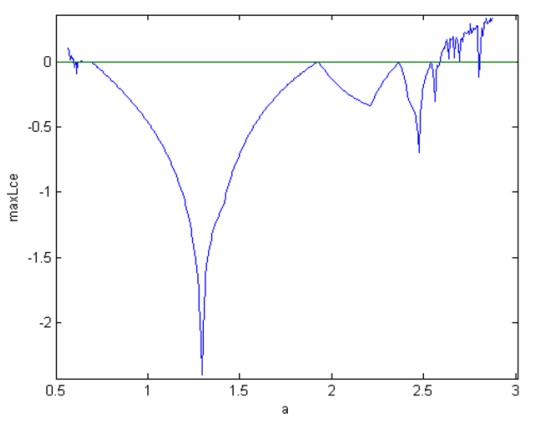

b
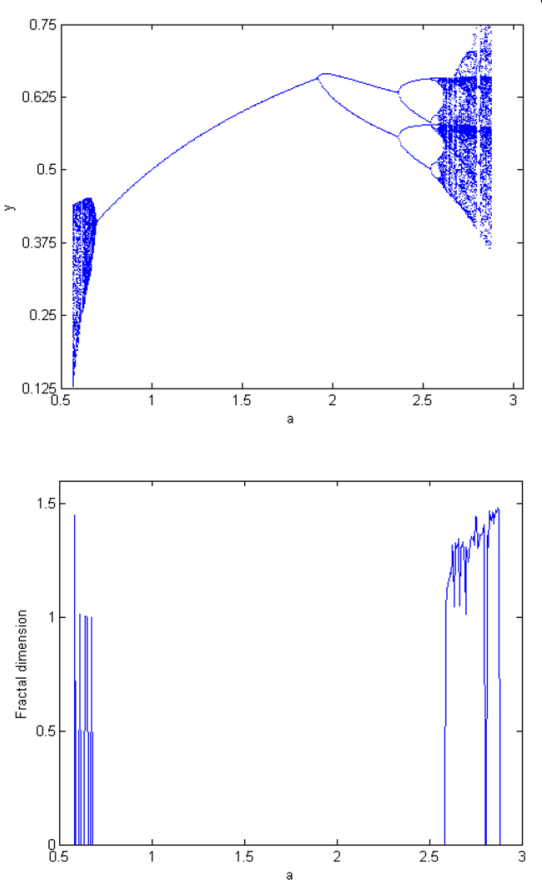

d

Fig. 4 NS-flip bifurcation of system (3). a Bifurcation for prey, b bifurcation for predator, $\mathbf{c}$ maximum Lyapunov exponents related to $\mathbf{a}, \mathbf{b}$. d FD associated with $\mathbf{a}$. $\left(x_{0}, y_{0}\right)=(0.37,0.37)$

Suppose parameters are considered as given in case (iv). When two more parameters change through its critical values, then system (3) can exhibit complex dynamic behavior. Under this parametric condition, the $3 D$ bifurcation diagrams in $(\delta, a, x)$-space are displayed in Fig. 5 a. Figure $5 b$ shows the $2 D$ projected maximum Lyapunov exponents onto $(\delta, a)$ plane. It is now easy to determine values of bifurcation parameters to see how does the dynamics of system (3) change from non-chaotic state to periodic or chaotic state. For instance, unstable chaotic trajectories appear in the system for parameters $\delta=4.15, \quad a=0.6$ whereas stable trajectories appear for $\delta=3.6, \quad a=0.6$ (see Fig. 3), which are conformable with the signs presented in Fig. 5b. It is also remarkable from Fig. 5 a that with the growth of parameter a, the predator-prey system (3) experiences NS bifurcation first and then flip bifurcation, and in between there is a stable window appears showing that the predator and prey coexist at a oscillatory balance behavior.

\section{Fractal dimension}

In order to characterize the strange attractors exists in a system, one can measure fractal dimensions (FD) [21, 22] which is defined by

$$
d_{L}=j+\frac{\sum_{i=1}^{j} h_{i}}{\left|h_{j}\right|}
$$

where $h_{1}, h_{2}, \ldots, h_{n}$ are Lyapunov exponents and $j$ is the largest integer such that $\sum_{i=1}^{j} h_{i} \geq$ 0 and $\sum_{i=1}^{j+1} h_{i}<0$.

For system (3), the fractal dimension $d_{L}$ takes the form 


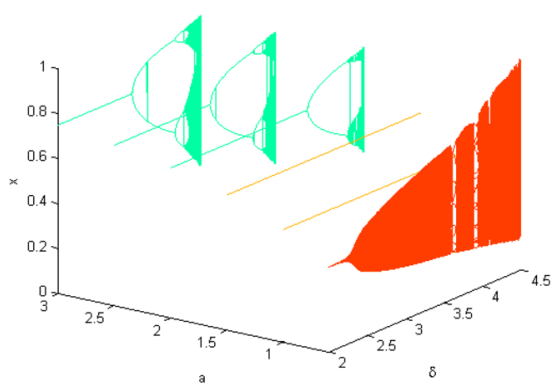

a

Fig. 5 Bifurcation diagram in $(\delta-a-x)$ space. (a) the transition between NS bifurcation and flip bifurcation for prey when $\delta \in[2.0,4.5], a=0.6,1.0,1.5,2.0,2.5,3.0 \in[0.6,3.0]$. b The 2D projection of 3D maximum Lyapunov exponents onto $(\delta, a)$ plane. $\left(x_{0}, y_{0}\right)=(0.37,0.37)$

$$
d_{L}=1+\frac{h_{1}}{\left|h_{2}\right|}, \quad h_{1}>0>h_{2} \quad \text { and } \quad h_{1}+h_{2}<0
$$

Considering parameter values as given in case (ii), FD of system (3) is plotted in Fig. 2e. The strange attractors of system (3) (see Fig. 3) and its corresponding FD (see Fig. 2e) confirms that growth of parameter $\delta$ causes a chaotic dynamics for the discrete-time ratiodependent Holling-Tanner system.

\section{Controlling chaos}

We will apply state feedback control method [20] to control chaos exists in system (3) at the state of unstable trajectories. By adding a feedback control law as the control force $u_{n}$ to system (3), the controlled system becomes

$$
\begin{aligned}
& x_{n+1}=x_{n}+\delta x_{n}\left[\left(1-x_{n}\right)-\frac{y_{n}}{x_{n}+a y_{n}}\right]+u_{n} \\
& y_{n+1}=y_{n}+\delta y_{n}\left[d\left(b-\frac{y_{n}}{x_{n}}\right)\right]
\end{aligned}
$$

and

$$
u_{n}=-k_{1}\left(x_{n}-x^{*}\right)-k_{2}\left(y_{n}-y^{*}\right)
$$

where the feedback gains are denoted by $k_{1}$ and $k_{2}$ and $\left(x^{*}, y^{*}\right)$ represent coexistence fixed point of system (3).

The Jacobian matrix $J_{c}$ of the controlled system(15) is

$$
J_{c}\left(x^{*}, y^{*}\right)=\left(\begin{array}{cc}
j_{11}-k_{1} & j_{12}-k_{2} \\
j_{21} & j_{22}
\end{array}\right)
$$

where $j_{11}=1+\delta\left(1-2 x+\frac{x y}{(x+a y)^{2}}-\frac{y}{x+a y}\right), j_{12}=\delta\left(\frac{a x y}{(x+a y)^{2}}-\frac{x}{x+a y}\right), j_{21}=\frac{d \delta y^{2}}{x^{2}}, j_{22}=$ $1-\frac{d \delta y}{x}+d \delta\left(b-\frac{y}{x}\right)$ are evaluated at $\left(x^{*}, y^{*}\right)$. The characteristic equation of $J_{c}\left(x^{*}, y^{*}\right)$ is

$$
\lambda^{2}-\left(\operatorname{tr} J_{c}\right) \lambda+\operatorname{det}_{c}=0
$$

where

$$
\begin{aligned}
\operatorname{tr} J_{c} & =j_{11}+j_{22}-k_{1}, \\
\operatorname{det} J_{c} & =j_{22}\left(j_{11}-k_{1}\right)-j_{21}\left(j_{12}-k_{2}\right) .
\end{aligned}
$$


Let $\lambda_{1}$ and $\lambda_{2}$ be the roots of (17). Then,

$$
\lambda_{1}+\lambda_{2}=j_{11}+j_{22}-k_{1}
$$

and

$$
\lambda_{1} \lambda_{2}=j_{22}\left(j_{11}-k_{1}\right)-j_{21}\left(j_{12}-k_{2}\right)
$$

The solution of the equations $\lambda_{1}= \pm 1$ and $\lambda_{1} \lambda_{2}=1$ determines the lines of marginal stability. These conditions confirm that $\left|\lambda_{1,2}\right|<1$. Suppose that $\lambda_{1} \lambda_{2}=1$, then from (19), we have

$$
l_{1}: j_{22} k_{1}-j_{21} k_{2}=j_{11} j_{22}-j_{12} j_{21}-1 .
$$

Now assume that $\lambda_{1}=1$, then from (18) and (19), we get

$$
l_{2}:\left(1-j_{22}\right) k_{1}+j_{21} k_{2}=j_{11}+j_{22}-1-j_{11} j_{22}+j_{12} j_{21} \text {. }
$$

Next, assume that $\lambda_{1}=-1$, then from (18) and (19), we obtain

$$
l_{3}:\left(1+j_{22}\right) k_{1}-j_{21} k_{2}=j_{11}+j_{22}+1+j_{11} j_{22}-j_{12} j_{21} \text {. }
$$

Then, the lines $l_{1}, l_{2}$, and $l_{3}$ (see Fig. 6a) in the $\left(k_{1}, k_{2}\right)$ plane determine a triangular region which keeps $\left|\lambda_{1,2}\right|<1$.

We have carried out numerical simulations to check how the implementation of feedback control method works and controls chaos at unstable state. Taking parameter values as in case (ii) with fixed $\delta=2.126$. We consider the feedback gains are as $k_{1}=-1.3$ and $k_{2}=0.16$ and initial value as $\left(x_{0}, y_{0}\right)=(0.65,0.95)$. Region of stable eigenvalues in $\left(k_{1}, k_{2}\right)$ plane is plotted in Fig. $6 \mathrm{a}$. We numerically show that at the fixed point $(0.662032,0.993048)$, the chaotic trajectory is stabilized, see Fig. $6 \mathrm{~b}, \mathrm{c}$.

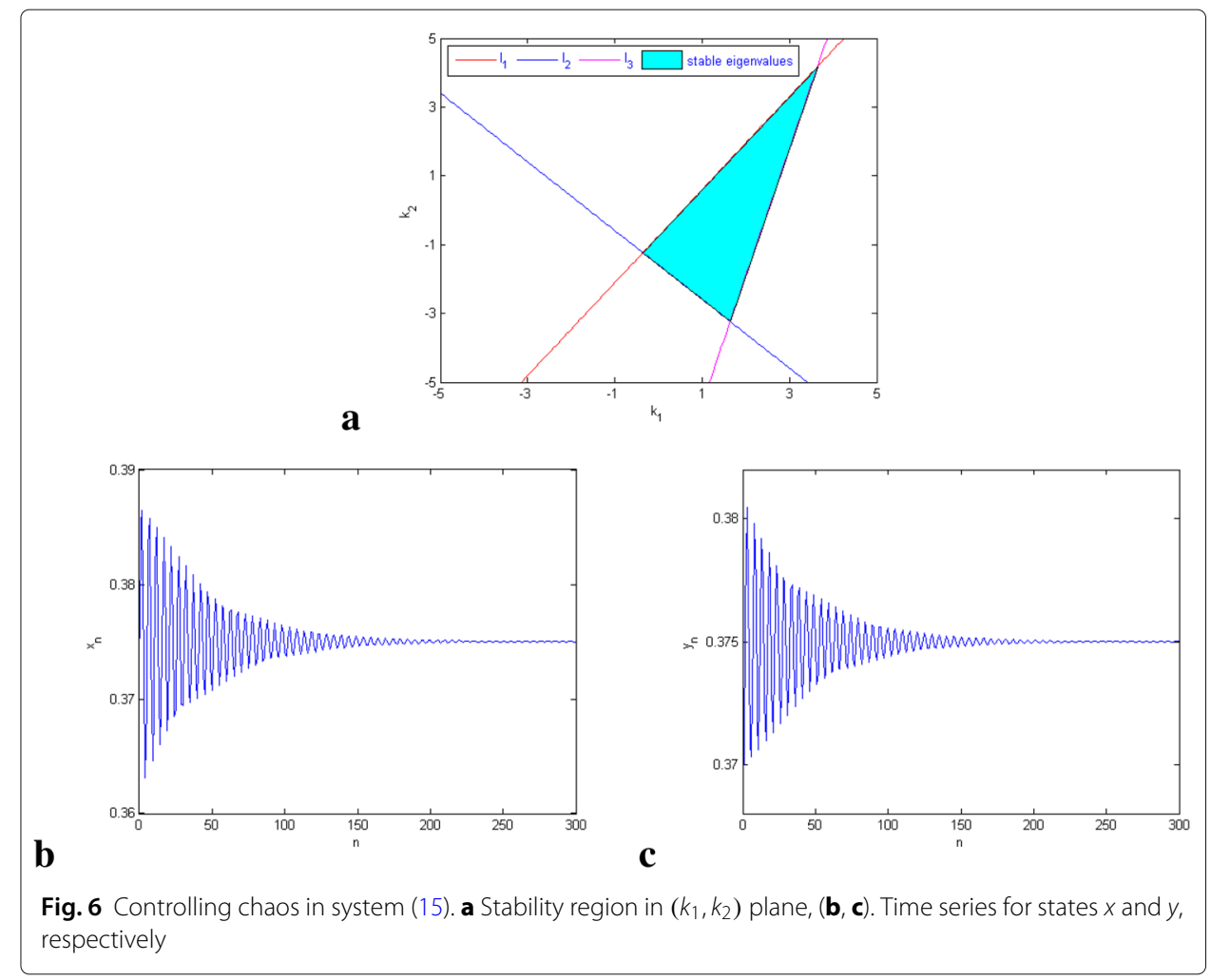




\section{Discussions}

This work is concerned with the dynamics and chaos control of a discrete-time ratiodependent Holling-Tanner model in $\mathbb{R}_{+}^{2}$. By the center manifold theory, we determine the existence condition and direction of flip and NS bifurcations of system (3) around coexistence fixed point. In particular, we show that system (3) experiences a flip or NS bifurcation at unique coexistence fixed point if parameter $\delta$ varies in the neighborhood of $\mathrm{FB}_{E_{2}}^{1}$ or $\mathrm{NSB}_{E_{2}}$. Based on Figs. 1 and 2, we notice that the small integral step size $\delta$ can stabilize the dynamical system (3), but the large integral step size may destabilize the system producing more complex dynamical behaviors. To see how does the integral step size play a key role in exploring the dynamical behaviors, we carry out numerical simulations to reveal unpredictable dynamics of the system including periods $-2,-4,-8,-12$, and -16 orbits via flip bifurcation and periods $-9,-10,-19$, and -38 orbits, invariant closed cycle, and chaotic sets via NS bifurcation, respectively. In addition, from Fig. 4, we can see that the appropriate choice of parameter $a$ can stabilize the dynamical system (3). However, for the low or high values of $a$ may destabilize system (3). Thus, with the increase of parameter $a$, it is shown that system (3) experiences NS and flip bifurcations both together. The two bifurcations cause the system to jump from steady state to chaotic dynamical behavior via periodic and quasi-periodic states and trigger routes to chaos, and vice-versa; that is, chaotic dynamics appear or disappear along with the emergence of bifurcations.

Moreover, in 3D bifurcation diagrams, we observe a dynamic transition between NS bifurcation and flip bifurcation. Through the two-dimensional parameter-spaces, we also notice that system dynamics can be periodic, quasi-periodic and chaotic. These complex dynamic behaviors of populations occurred by the flip and NS bifurcations can be explained ecologically, that is, the predator and prey population densities can fluctuate at regular or irregular intervals or the predator-prey system become unstable [23, 24]. We can also say that when the prey population are abundant, the consumption of prey by predator may have a marginal effect on the dynamics of prey. The presence of chaos is verified by the sign of maximum Lyapunov exponents and fractal dimension. Finally, we provide state feedback control method to control chaos at unstable trajectories. We would expect to explore more analytical results on multiple-parameter bifurcation of the system in future.

Acknowledgements

The author would like to thank the editor and the referees for their valuable comments and suggestions, which led to the improvements of the paper.

Author's contributions

The author carried out the proof of the main results and approved the final manuscript.

Funding

Not applicable.

Availability of data and material

Not applicable.

Ethics approval and consent to participate

Not applicable.

Consent for publication

Not applicable.

Competing interests

The authors declare that there is no competing interests. 
Received: 27 June 2019 Accepted: 5 November 2019

Published online: 28 November 2019

\section{References}

1. Li, Y., Xiao, D.: Bifurcations of a predator-prey system of Holling and Leslie types. Chaos Solit. Fract. 34(2), 606-620 (2007). https://doi.org/10.1016/j.chaos.2006.03.068

2. Hsu, S. B., Hwang, T. W.: Global stability for a class of predator-prey systems. SIAM J. Appl. Math. 55, 763-783 (1995)

3. Gasull, A., Kooij, R. E., Torregrosa, J.: Limit cycles in the Holling-Tanner model. Publ. Mat. 41, 149-167 (1997)

4. Liang, Z., Pan, H.: Qualitative analysis of a ratio-dependent Holling-Tanner model. J. Math. Anal. Appl. 334, 954-964 (2007)

5. He, Z. M., Lai, X.: Bifurcation and chaotic behavior of a discrete-time predator-prey system. Nonlinear Anal. Real World Appl. 12, 403-417 (2011)

6. He, Z. M., Li, B.: Complex dynamic behavior of a discrete-time predator-prey system of Holling-III type. Adv. Differ. Equ. 180 (2014)

7. Rana, S. M. S.: Bifurcation and complex dynamics of a discrete-time predator-prey system with simplified Monod-Haldane functional response. Adv. Differ. Equ. 345 (2015). https://doi.org/10.1186/s13662-015-0680-7

8. Rana, S. M. S.: Chaotic dynamics and control of discrete ratio-dependent predator-prey system. Discret. Dyn. Nat. Soc., 1-13 (2017). https://doi.org/10.1155/2017/4537450

9. Rana, S. M. S., Kulsum, U.: Bifurcation analysis and chaos control in a discrete-time Predator-Prey System of Leslie Type with Simplified Holling Type IV Functional Response. Discret. Dyn. Nat. Soc. (2017). https://doi.org/10.1155/ 2017/9705985

10. Rana, S. M. S.: Bifurcations and chaos control in a discrete-time predator-prey system of Leslie type. J. Appl. Anal. Comput. 9(1), 31-44 (2019). https://doi.org/10.11948/2019.31

11. Tan, W., Gao, J., Fan, W.: Bifurcation Analysis and Chaos Control in a Discrete Epidemic System. Discret. Dyn. Nat. Soc. (2015). https://doi.org/10.1155/2015/974868

12. Zhao, M., Xuan, Z., Li, C.: Dynamics of a discrete-time predator-prey system. Adv. Differ. Equ. 191 (2016). https://doi. org/10.1186/s13662-016-0903-6

13. Zhao, M., Li, C., Wang, J.: Complex dynamic behaviors of a discrete-time predator-prey system. J. Appl. Anal. Comput. 7(2), 478-500 (2017). https://doi.org/10.11948/2017030

14. Liu, W., Cai, D.: Bifurcation, chaos analysis and control in a discrete-time predator-prey system. Adv. Differ. Equ. 11 (2019)

15. Kangalgil, F.: Neimark-Sacker bifurcation and stability analysis of a discrete-time prey-predator model with Allee effect in prey. Adv. Differ. Equ. 92 (2019)

16. Hu, D. P., Cao, H. J.: Bifurcation and chaos in a discrete-time predator-prey system of Holling and Leslie type. Commun. Nonlinear Sci. Numer. Simulat. 22, 702-715 (2015)

17. Cao, H., Yue, Z., Zhou, Y.: The stability and bifurcation analysis of a discrete Holling-Tanner model. Adv. Differ. Equ. $330(2013)$

18. Zhao, J., Yan, Y.: Stability and bifurcation analysis of a discrete predator-prey system with modified Holling-Tanner functional response. Adv. Differ. Equ. 402 (2018)

19. Kuzenetsov, Y. A.: Elements of Applied Bifurcation Theory, 2nd Ed. Springer-Verlag (1998)

20. Elaydi, S. N.: An Introduction to Difference Equations. Springer-Verlag, New York (2005)

21. Cartwright, J. H. E.: Nonlinear stiffness Lyapunov exponents and attractor dimension. Phys. Lett. A. 264, 298-304 (1999)

22. Kaplan, J. L., Yorke, Y. A.: A regime observed in a fluid flow model of Lorenz. Commun. Math. Phys. 67, 93-108 (1979)

23. Hastings, A., Hom, C. L., Ellner, S., Turchin, P., Godfray, H. C. J.: Chaos in ecology: Is mother nature a strange attractor?. Ann. Rev. Ecol. Evol. Syst. 24, 1-33 (1993)

24. Nicholson, A. J.: The self-adjustment of populations to change. Cold Spring Harb. Symp. Quant. Biol. 22, 153-173 (1957)

\section{Publisher's Note}

Springer Nature remains neutral with regard to jurisdictional claims in published maps and institutional affiliations.

\section{Submit your manuscript to a SpringerOpen ${ }^{\circ}$ journal and benefit from:}

- Convenient online submission

Rigorous peer review

Open access: articles freely available online

- High visibility within the field

- Retaining the copyright to your article

Submit your next manuscript at $\gg$ springeropen.com 